\section{Evidence-based primary care} approach to treating people with COVID-19 infection to prevent life-threatening complications: A review of the evidence for practical
application in a clinical setting

\author{
Thomas A Sharon* \\ Adult and Gerontological Primary Care Nurse Practitioner, Las Vegas, Nevada 89148, USA
}

\section{Abstract}

The NIH has published treatment guidelines for treating COVID-19 patients in the hospital. However, as of this writing, there are no established protocols for treating COVID-19 positive patients in primary care. Accordingly, this investigator has taken on the task of reviewing the medical literature to be able to propose evidence-based protocols for treating COVID-19 positive patients in primary care. The CDC is advising people to do nothing when they find out they are positive for COVID-19 unless they have symptoms.

The evidence from the literature irrefutably shows COVID-19 infection evokes a massive and deadly hyperinflammatory response called the "Cytokine storm" and that Cytokine levels in the blood have a predictive value in identifying an impending Cytokine storm. With such data primary care providers can effectively lower Cytokine levels and prevent critical illness and death.

Accordingly, this paper presents identification of the problem of not having standard practices in primary care for people who are positive for COVID-19 and not knowing who is at risk. Moreover, the evidence shows that knowing vitamin $D$ levels and correcting deficiencies can go a long way in reducing Cytokine levels. Additionally, the literature review presents evidence that undeniably shows the stark possibility that many of the COVID-19 related deaths can be prevented by identifying who is at risk for the Cytokine storm and other complications and providing early treatment even before symptoms appear.

\section{More Information}

*Address for Correspondence: Thomas A Sharon, DNP, MPH, APRN-BC, Adult and Gerontological Primary Care Nurse Practitioner, 9573 Ancala Hollow Ct, Las Vegas, Nevada 89148, Tel: 702-209-5648; Email: nursetom@msn.com

Submitted: January 19, 2021

Approved: March 15, 2021

Published: March 16, 2021

How to cite this article: Sharon TA. Evidencebased primary care approach to treating people with COVID-19 infection to prevent life-threatening complications: A review of the evidence for practical application in a clinical setting. Clin J Nurs Care Pract. 2021; 5: 009-014.

DOI: 10.29328/journal.cjncp.1001031

Copyright: @ 2021 Sharon TA. This is an open access article distributed under the Creative Commons Attribution License, which permits unrestricted use, distribution, and reproduction in any medium, provided the original work is properly cited.

Keywords: COVID-19; Cytokines; Cytokine storm; inflammation; Vitamin D; Blood clots; Platelets; D-dimer; Pneumonia; Pulmonary embolism

(D) Check for updates

(6) OPEN ACCESS

\section{Introduction}

The Centers for Disease Control and Prevention (CDC), through their website, continues to publish outdated messages to people who test positive for COVID-19 [1]. Their public advice needs to be revised and updated in the face of massive amounts of new research findings appearing in the medical literature daily since April 2020 (see literature review).

"Most people with COVID-19 have mild illness and can recover at home without medical care.

"When to seek emergency medical attention:
"Look for emergency warning signs* for COVID-19. If someone is showing any of these signs, seek emergency medical care immediately:

- Trouble breathing

- Persistent pain or pressure in the chest

- New confusion

- Inability to wake or stay awake

- Bluish lips or face"

In effect, the CDC is telling people they do not need inhospital medical attention unless they have life threatening 
symptoms. Although the statistical evidence supports the conclusion that most people can survive without medical intervention, the problem is too many people cannot. Once such symptoms as dyspnea, persistent chest pain, stuporous mental status, delirium, and facial cyanosis appear requiring emergency hospitalization, fatality rates as high as $50 \%$ have been reported (Joyner, Bruno, Klaasen et al, 2020). Therefore, notwithstanding that when the pandemic first arrived in February 2020 there was little choice for lack of medical knowledge, waiting at home in quarantine until symptoms appear before calling a medical provider should now be considered unwise and dangerous.

The brief medical literature review that follows will demonstrate that all people who test positive for COVID-19, need a series of blood tests to predict whether they are at risk for a cascade of complications such as adult respiratory distress syndrome, cardiomyopathy, coronary embolism, pulmonary embolism, embolic stroke, and kidney failure (to name a few). Being told that most people can recover without medical attention does nothing to save lives. However, in the face of a death toll of more than 250,000 in the USA alone at the time of this writing, finding out who is at risk of such lethal complications can save tens of thousands.

\section{Literature review for evidence synthesis}

\section{The devastating effects of cytokine storm}

Kaur, Bansal, Kollimuttathuillam, et al. [2] conducted a systemic review of 82 articles containing retrospective analyses of clinical data of patients treated in hospitals worldwide. The data covered etiology, mode of transmission and pathophysiology of the SARS-Cov-2 virus, hematological abnormalities leading to thromboembolytic disease, and Cytokine storm causing massive systemic inflammation. These authors' review of the literature provides substantial evidence of identifying the cascade of multiple organ failures that occur. Additionally, they have offered specific diagnostic protocols to identify those at risk of such life-threatening complications before they arise. Thus, primary care providers have an opportunity to initiate preventive pharmacotherapy.

The data collection in the review confirms COVID-19 human to human transmission occurs via respiratory droplets and close contact. This virus can survive 3 hours in the air, 4 hours on a copper surface, 24 hours on cardboard, and 72 hours on plastic or stainless steel. The data also reveals viral load and transmission is the same for symptomatic and asymptomatic patients. Moreover, we also learn that on a cellular level COVID-19 virus has an affinity for angiotensinconverting enzyme-2 (ACE-2) receptors which are in the respiratory tract, cardiac, digestive tract, and urinary tract, making all vital organ systems potentially susceptible to viral attack at the cellular level.

The hematology laboratory findings in the review present an interesting array of prognostic information. The authors reviewed a meta-analysis involving lymphopenia (T lymphocyte depletion), thrombocytopenia, leukopenia, leukocytosis (in fewer cases), and D-dimer elevations indicating elevated risk of thrombus formation. The data suggests prevalence of such abnormalities in critically ill patients, while the platelet count seems to offer predictive findings for impending critical illness response. Concomitantly, arterial thrombosis is associated with COVID-19 complications resulting in myocardial infarction, pulmonary embolism, and embolic stroke.

In reviewing the pathophysiology of arterial thrombus development, the authors mentioned the COVID-19 related cytokine storm as a major factor in the COVID-19 hyperinflammatory response. Cytokines result in severe systemic endothelial damage which can lead to adult respiratory distress syndrome (ARDS), hypercoagulation, and disseminated intravascular coagulation (DIC). They postulated the DIC could be part of the main pathophysiological response of COVID-19 infection. Cytokine release syndrome (CRS) is a systemic hyper response to infections via the ACE2 receptors and binding with alveolar cells in the lungs. These researchers came to view the cytokine storm as a major cause of COVID-19 related death and more importantly, the elevations in cytokines can be detected before the critical illness develops and corresponds to elevations in D-dimer levels. Since the COVID-19 related CRS becomes an autoimmune disease, whereby the immune system attacks the cells of the lungs and other vital organs, these authors favor aggressive treatment with Tocilizumab, an immunosuppressant drug used to treat rheumatoid arthritis. The authors cited a Chinese study which showed favorable results in $78 \%$ of patients treated. There are also other studies cited showing promising outcomes with dexamethasone.

Liu, Zhang, Huang, et al. [3] used the Bio-Plex Pro Human Cytokine Screening Panel to measure levels of 48 key cytokines/chemokines in blood plasma in 12 COVID-19 patients who were classified as having mild and severe symptoms, in comparison with well individuals.

Using Spearman's rank coefficient correlation analysis, these authors discovered an association between 15 of the 48 Cytokines observed and the COVID-19 viral load in affected patients. This data suggests that those 15 Cytokines (IL-12, IFN- $\gamma$, IL-2, HGF, IFN- $\alpha 2$, IL-4, IL-17, IP-10, G-CSF, IL-10, IL-1ra, M-CSF, IL-1 $\alpha$ and IL-7) are early biomarkers for disease severity with people who test positive for COVID-19 infection.

Additionally, the authors claim to have demonstrated that a Cytokine storm is a likely occurrence with COVID-19 infection. These researchers concluded that blood levels of 15 of the aforementioned Cytokines should be obtain and Cytokine suppressors should be administered as soon as 
possible. They have classified three levels of severity of infection as follows:

1. Mild infection: fever, respiratory congestion, coughing, and chest X-ray showing infiltrates.

2. Moderate infection: fever, respiratory congestion, coughing, chest X-ray showing infiltrates, Respiration rate $\geq 30 / \mathrm{min}$, resting oxygen saturation $\leq 93 \%$, and/ or $\mathrm{FiO}_{2} \leq 300 \mathrm{mmHg}$.

3. Severe infection: Respiratory failure requiring mechanical ventilation, shock and/or failure of other organs requiring critical care.

These authors provided further evidence that Cytokine levels, other inflammation markers and lung imaging should be obtained as early as possible with treatment rendered in accordance with the results and the presenting symptoms.

Ragab, Salah, Taeimah, et al. [4] reviewed 30 articles containing retrospective clinical and epidemiological data. They confirmed the mode of transmission and mortality rates across the board. They concluded from the literature that both derivatives of COVID-19 (SARS-CoV and MERS$\mathrm{CoV}$ ) cause massive production of Cytokines known as the "cytokine storm" in an auto immune-like syndrome that if unchecked results in a cascade of multiple organ failure starting with the lungs. Elevated levels of IL-1b, IL-7, IL-8, IL9, IL-10, FGF, G-CSF, GM-CSF, IFN-g, IP-10, MCP-1, MIP-1A, MIP1-B, PDGF, TNF-a, and VEGF show up early on before the disastrous irreversible effects occur. Therefore, the timefactor for measuring Cytokine levels is critical in preventing hospitalization and death.

Fara, Mitrev, Rosalia, et al. [5] identified the progression of complications arising from COVID-19 infections as follows:

Onset

1. Fever

2. Dry cough

3. Myalgia

4. Fatigue

5. Sputum production

6. Headache

7. Hemoptysis

8. Diarrhea

Progression

1. Pneumonia with ARDS

2. Acute cardiac injury
3. Secondary bacterial pneumonias

4. Pulmonary embolism

5. Embolic stroke

These authors studied the cytokine storm and its debilitating effects. They explained that an infected cell, activates large numbers of white blood cells, including $B$ cells, T cells, natural killer cells, macrophages, dendritic cells, and monocytes through receptor-ligand interactions, which results in a release of inflammatory cytokines, activating more white blood cells in a positive feedback loop. We learn that classic signs of inflammation can be seen with heat, pain, erythema, edema, and loss of tissue function. Although inflammation is a natural process that results in healing for the most part, when healing occurs with fibrosis it can result in persistent organ malfunction. In other words, the COVID-19 virus leaves a residual genetic signature in the invaded cells causing a continuous hyperinflammation catalyzed by the Cytokine storm.

These authors extrapolated from their literature review that the inflammatory response to COVID-19 also contains secondary hemophagocytic lymphohistiocytosis (HLH) as additional components leading to hepatomegaly and splenomegaly. A risk algorithm may be employed to assess the likelihood of developing HLH. These investigators also concluded that the Interluken-6 (Il-6) Cytokine becomes elevated early in the infection process and thus has predictive attributes in assessing the risk of Cytokine storm. These authors provided further evidence of the need to obtain Cytokine blood levels early on and treat elevated levels with steroids or other immunosuppressive agents.

Lu, Zhang, Zhan, et al. [6], recognized that focusing on nullifying the cytokine storm (CS) is a vital primary care obligation. They investigated the question of which cytokine to target in this effort. These authors also recognized that the failure to address CS can lead to acute respiratory distress syndrome (ARDS), cardiomyopathy, myocardial infarction, disseminated intravascular coagulation (DIC), neurological impairment, gastrointestinal tract, and/ or kidney dysfunction, resulting in high mortality. They recognized from their literature review that COVID-19 causes rapid onset and prolonged blood elevation of excessive amounts of cytokines such as interleukin (IL)-6, IL-8, tumornecrosis factor (TNF)-a, interferon (IFN)-g, and chemokines. Accordingly, Lu, et al. [6] reviewed the literature of clinical trials of various pharmacological therapies in reducing the autoimmune devastation. They concluded that knowledge of the immunological pathways leading to COVID-19 deaths and an efficacious strategy to reduce and nullify the CS were lacking. Accordingly, Lu, et al. [6] proposed such strategies to target the culpable immunologic pathways to save lives.

These authors alluded to the $20+$ different cytokines 
that become elevated, making effective intervention difficult. Upon further review of newly published research, they concluded that the fatality of COVID-19 is due to one Cytokine: Interluken-6 (IL-6). Consequently, IL-6 should be a main target for Cytokine reduction. However, these investigators noted that IL- 6 does not appear early in the COVID-19 disease process and thus it has no predictive value in determining who is at risk. Consequently, they reported that elevations of plasma levels of IP-10 and MCP-3, appear early and can function as predictors of disease severity and fatal outcomes.

Therefore, Lu, et al. [6] proposed their theory of three stages of immunopathological pathway to COVID-19 mortality: initiation, amplification, and consummation.

The initiation phase of COVID-19 is presumably a rapid replication of SARS-CoV- 2 with the initial massive production of chemokines in the lung tissues. The infection-induced chemokines then begin to recruit intrinsic and adaptive immune cells to destroy infected lung tissue.

The amplification phase starts at day 5-14 after onset of infection in fatal COVID-19 cases. During this second phase, the disease progresses by amplification of inflammatory autoimmune responses resulting in rapid progression to critical condition. Patients first display severe symptoms, including productive cough and dyspnea within 7 days of onset followed by sepsis within 10 days and ARDS within 12 days requiring admission to ICU. The authors described the second phase as a "rapid deterioration of lung function, likely caused by large-scale inflammatory infiltration induced damage."

The third phase as described starts on the $15^{\text {th }}$ day ending in death. This rapid deterioration is a cascade of organ failures caused by the CS. Intubation with mechanical ventilation is necessary to prolong life. The authors allude to clinical evidence showing further rise in Cytokines lead by IL-6.

Lu, et al. [6] suggested the administration of Anakinra, an IL-1 receptor antagonist used as an immunosuppressant drug, to stem the tide of CS. This medication, like Tocilizumab, has been used to treat rheumatoid arthritis. These authors cited 2 studies that showed substantial reduction in death treating severe COVID-19 illness with Anakinra.

These authors also proposed strategies to target CS to prevent COVID-19 related death. They concluded that the clinical efficacy of focusing on IL-6 blockade is suboptimal. Efficacious treatment strategy requires blocking production of the 20 Cytokines mentioned above. These authors cited a review of clinical trials targeting 10 of the Cytokine culprits. They concluded from recently published studies that the Chemokines CCL2, CCL3, CCL5, and IP10 are the prime targets for blocking strategies which starts with timeliness. They strongly suggested early treatment within the first 4 days of onset during the initiation phase with a combination of Cytokine inhibitors, since various immunosuppressant drugs have shown promising results in preventing death.

According to the National Institute of Health (NIH), Remdesivir is the only FDA-approved antiviral drug for COVID-19 infection [7]. The NIH divides the treatment of COVID-19 infection into two components: 1) antiviral therapies, and 2) immunosuppressive/anti-inflammatory therapies. The NIH recommends early intervention with the former and the latter should be reserved for later stages. There are three problems with this therapeutic guideline. First, Remdesivir is only available in the hospital so primary care patients outside the hospital have no access to this drug in either intravenous or oral form.

Second, the evidence presented in this review overwhelmingly points to providing immunosuppressive/ anti-inflammatory therapies as early as possibly to patients who have elevated Cytokine levels regardless of whether there are symptoms or not.

Finally, Remdesivir, in a randomly controlled trial, was shown to merely shorten recovery time in hospitalized patients, but the treatment group still had a $6.7 \%$ mortality rate among infected patients. Thus, there is no evidence to show that Remdesivir or any other known antiviral is efficacious in eradicating the COVID-19 virus [8].

Furthermore, the guidelines offered by the NIH pertain to hospitalized COVID-19 patients. A search of the NIH website did not reveal any guideline specific for treating COVID-19 positive patients in an outpatient setting. Although, the NIH does address the advent of the Cytokine storm with the devastating effects of hyperinflammation, the recommendations of using steroids and immunosuppressant agents such as Bamlanivimab and the Casirivimab Plus Imdevimab Combination pertains again to hospitalized patients. Additionally, those agents are available on an emergency use authorization only and, as of this writing, they have not been approved by the FDA for COVID-19 treatment.

\section{Vitamin d deficiency and cytokine storm syndrome}

Rhodes, Subramanian, Laird, et al. [9] reviewed the literature to determine the link between vitamin D deficiency and the severity of the hyperinflammatory response to COVID-19 infection. They noted the wide variety of prognoses from being asymptomatic to dying of adult respiratory distress syndrome (ARDS), aberrant blood clots, or vital organ failure. They also observed from the reviewed data that there was a geographical differential in the morbidity and mortality rates of COVID-19 infection. Areas closer to the equator had lower rates than areas further away. The extrapolated the theory of the former having more direct sunlight and thus 
had exposure to higher ultraviolet (UV) radiation levels. UV radiation is known to synthesize vitamin $D$ through the skin. Thus, vitamin D deficiency is more prevalent in areas with less exposure to UV radiation. From experimental data in these authors' review, they surmised that vitamin D increases the ratio of angiotensin-converting enzyme 2 (ACE2) to ACE, which causes an increase in angiotensin II hydrolysis thereby reducing subsequent inflammatory cytokine response to pathogens and lung injury. Therefore, knowing the vitamin D blood levels in COVID-19 positive patients is a vital part of assessing risk of critical illness and death.

\section{Discussion}

As of this writing, there are no generally accepted treatment guidelines for treatment of COVID-19 positive patients in a primary care setting. The evidence found in this review undeniably points to early intervention with diagnostic testing to know who is at risk for an impending Cytokine storm. Therefore, this writer recommends the following for diagnosing existing or potential hyperinflammatory responses to COVID-19 infection:

Laboratory testing:

1. CMP to get a baseline for electrolytes and kidney and liver function;

2. Lipid panel to get a baseline of heart disease risk factors;

3. $\mathrm{CBC}$ with differential and platelets to determine the risk of forming aberrant blood clots;

4. Assay for cytokine panel 12 protocol to determine if there are elevations to indicate an impending Cytokine storm;

5. D-dimer to determine the presence or risk of thrombus formation in arteries and veins;

6. PT, PTT and INR to determine clotting time;

7. Vitamin D blood level

8. Chest X-ray to determine the presence or absence of infiltrates.

Treatment for asymptomatic COVID-19 positive patients:

1. If all laboratory values and chest X-rays are within normal limits, the patient requires only medical watch with follow up phone calls at reasonable intervals.

2. For elevations in Cytokines levels steroid therapy should be initiated and maintained until Cytokine levels are normal:

a. Prednisone $20 \mathrm{mg}$ daily, or

b. Methylprednisolone $4 \mathrm{mg}$ twice per day, or c. Dexamethasone - Initial dose: 5 mg daily.

d. Dosages will have to be titrated depending on the Cytokine level responses to steroid therapy

e. Patients should be monitored as needed for symptoms and vital signs

Treatment for symptomatic COVID-19 positive patients:

1. For respiratory symptoms:

a. Prednisone $20 \mathrm{mg}$ daily, or

b. Methylprednisolone $4 \mathrm{mg}$ twice per day, or

c. Dexamethasone - Initial dose: $5 \mathrm{mg}$ daily.

d. Dosages will have to be titrated depending on the Cytokine level responses to steroid therapy.

e. Albuterol sulfate $0.63 \mathrm{mg} / 3 \mathrm{ml}$ or $1.25 \mathrm{mg} / 3 \mathrm{ml}$ solution for nebulization, inhale $3 \mathrm{ml} \mathrm{q6h}$ PRN for wheezing, tightness in the chest or difficulty breathing.

f. Budesonide $0.25 \mathrm{mg} / 2 \mathrm{ml}, 0.5 \mathrm{mg} / 2 \mathrm{ml}$, or $1 \mathrm{mg} / 2$ $\mathrm{ml}$ suspension for nebulization twice per day for 30 days.

g. ProAir HFA $90 \mathrm{mcg} /$ actuation aerosol inhaler 2 puffs as needed q6h (use when the nebulizer is unavailable).

h. Symbicort $80 \mathrm{mcg}-4.5 \mathrm{mcg} / \mathrm{actuation}$ or $160 \mathrm{mcg}$ $4.5 \mathrm{mcg} /$ actuation HFA aerosol inhaler 2 puffs twice per day (use when the nebulizer is unavailable).

i. Zyrtec-D $5 \mathrm{mg}-120 \mathrm{mg}$ tablet, extended release 1 daily for nasal and chest congestion.

j. Promethazine $6.25 \mathrm{mg}$-codeine $10 \mathrm{mg} / 5 \mathrm{~mL}$ syrup, $5 \mathrm{ml}$ as needed for cough q6h.

k. If infiltrates are present on chest X-ray then administer antibiotic for assumed secondary bacterial infection. The usual choices are Azithromycin, Augmentin, Bactrim, and Levaquin.

\section{Conclusion}

The CDC informs the public the most people recover from COVID-19 without medical intervention. The COVID-19 disease onset is insidious and unpredictable. $20 \%$ of infected people have moderate to severe symptoms and $80 \%$ have mild or no symptoms. The problem is that the length of time from infection to symptoms can vary from one person to another, so people who are positive will stay home until symptoms appear. Since the CDC advisory tells people to seek medical attention only when they are experiencing 
dyspnea and hypoxia, by the time infected people reach the hospital emergency department the chance of survival drops to $50 \%$ at the hospital door and $30 \%$ in the ICU. By that time the Cytokine storm has wreaked havoc with the lungs and other vital organs.

Therefore, this author proposes that primary care providers begin medical management as suggested above at the time that a person is first found to be COVID-19 positive to find out who is at risk for the deadly response of hyperinflammation and coagulation abnormalities. Being told that most people survive without medical management does nothing to save lives. Finding out who is at risk for deadly complications will save many thousands.

\section{References}

1. Center for Disease Control and Prevention website. 2020. https://www.cdc.gov/coronavirus/2019-ncov/if-you-are-sick/stepswhen-sick.html.

2. Kaur S, Bansal R, Kollimuttathuillam S, Gowda AM, Singh B, et al. The looming storm: Blood and cytokines in COVID-19. Blood Rev. 2020; 100743.

PubMed: https://pubmed.ncbi.nIm.nih.gov/32829962/

3. Liu $Y$, Zhang $C$, Huang $F$, Yang $Y$, Wang $F$, et al. Elevated plasma level of selective cytokines in COVID-19 patients reflect viral load and lung injury. National Sci Rev. 2020.

4. Ragab D, Salah Eldin H, Taeimah M, Khattab R, Salem R. The COVID-19 cytokine storm; what we know so far. Frontiers Immunol. 2020; 11: 1446.

5. Fara A, Mitrev Z, Rosalia RA, Assas BM. Cytokine storm and COVID-19: a chronicle of pro-inflammatory cytokines. Open Biol.
2020; 10: 200160.

PubMed: https://pubmed.ncbi.nlm.nih.gov/32961074/

6. Lu L, Zhang $\mathrm{H}$, Zhan M, Jiang J, Yin $\mathrm{H}$, et al. Preventing mortality in COVID-19 patients: which cytokine to target in a raging storm? Front Cell Dev Biol. 2020; 8: 677.

PubMed: https://www.ncbi.nlm.nih.gov/pmc/articles/PMC7379422/

7. National Institute of Health Website. Therapeutic Management of Patients with COVID-19. NIH Covid-19 Treatment Guidelines. 2020.https://www.covid19treatmentguidelines.nih.gov/therapeuticmanagement/

8. Beigel JH, Tomashek KM, Dodd LE, Mehta AK, Zingman BS, et al. Remdesivir for the Treatment of Covid-19-Final Report. [published online October 8, 2020]. New Engl J Med. 2020; 383: 1813-1826. PubMed: https://pubmed.ncbi.nlm.nih.gov/32445440/

9. Rhodes JM, Subramanian S, Laird E, Griffin G, Kenny RA. Perspective: Vitamin $D$ deficiency and COVID-19 severity-plausibly linked by latitude, ethnicity, impacts on cytokines, ACE2 and thrombosis (R1). J Intern Med. 2020.

PubMed: https://www.ncbi.nlm.nih.gov/pmc/articles/PMC7361294/

10. Han $\mathrm{H}$, Ma Q, Li C, Liu R, Zhao L, et al. Profiling serum cytokines in COVID-19 patients reveals IL-6 and IL-10 are disease severity predictors. Emerg Microbes Infect. 2020; 9: 1123-1130. PubMed: https://pubmed.ncbi.nlm.nih.gov/32475230/

11. Lammers $T$, Sofias $A M$, van der Meel R, Schiffelers R, Storm G, et al. Dexamethasone nanomedicines for COVID-19. Nat Nanotechnol. 2020; 15: 622-624.

PubMed: https://pubmed.ncbi.nlm.nih.gov/32747742/

12. Sang $Y$, Roest $M$, de Laat $B$, de Groot PG, Huskens D. Interplay between platelets and coagulation. Blood Rev. 2020. 100733. PubMed: https://www.ncbi.nlm.nih.gov/pmc/articles/PMC7354275/

13. Xu ZS, Shu T, Kang L, Wu D, Zhou X, et al. Temporal profiling of plasma cytokines, chemokines and growth factors from mild, severe and fatal COVID-19 patients. Signal Transduct Target Ther. 2020; 5: 100. 